\title{
Congenital Defect of the Toenail
}

Daniel W. Cole, MD; Tomas Huerta, MD; Jennifer B. Mancuso, MD

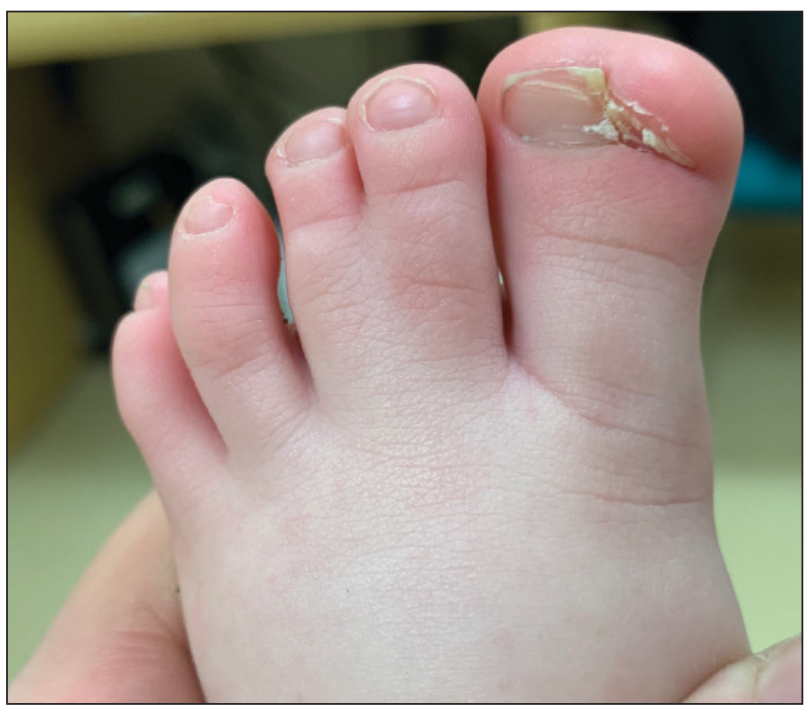

An 18-month-old girl presented for evaluation of nail dystrophy. The patient's parents stated that the left great toenail had been dystrophic since birth, leading to skin irritation and "snagging" of the toenail on socks and footwear. Additional history revealed that the patient also had webbed toes, and there was a paternal family history of polydactyly and syndactyly. Physical examination revealed webbing of the second and third toes to the distal interphalangeal joints on both feet, marked nail plate dystrophy on the left big toe, and an irregularly shaped nail plate on the right big toe. The patient had no similar findings on the hands.

\section{WHAT'S THE DIAGNOSIS?}
a. congenital hypertrophy of the lateral nail fold
b. congenital malalignment of the great toenails
c. fetal hydantoin syndrome (phenytoin use during pregnancy)
d. nail-patella syndrome (onycho-osteodysplasia; Fong disease)
e. onychodystrophy secondary to polydactyly

\footnotetext{
Dr. Cole is from the Wayne State University School of Medicine, Detroit, Michigan. Drs. Huerta and Mancuso are from the Department of Dermatology, University of Michigan, Ann Arbor. 


\section{THE DIAGNOSIS:}

\section{Onychodystrophy Secondary to Polydactyly}

$\longrightarrow$ adiographs of the feet demonstrated an accessory distal phalanx of the left great toe with a similar smaller accessory distal phalanx on the right great toe (Figure). The patient was referred to orthopedic surgery, and surgical intervention was recommended for only the left great toe given recurrent skin inflammation and nail complications. An excision of the left great toe polydactyly was performed. The patient healed well without complications.

Many clinically heterogeneous phenotypes exist for polydactyly and syndactyly, which both are common entities with incidences of 1 in 700 to 1000 births and 1 in 2000 to 3000 births, respectively. ${ }^{1}$ Both polydactyly and syndactyly can be an isolated variant in newborns or present with multiple concurrent malformations as part of a genetic syndrome, with more than 300 syndromic anomalies described. The genetic basis of these conditions is equally diverse, with homeobox genes, hedgehog pathways, fibroblast growth factors, and boneand cartilage-derived morphogenetic proteins implicated in their development. ${ }^{1}$
The differential diagnosis for our patient included congenital malalignment of the great toenails, nail-patella syndrome, onychodystrophy secondary to polydactyly, and congenital hypertrophy of the lateral nail fold. Given the strong family history, polydactyly was suspected.

Congenital malalignment of the great toenails results in lateral deviation of the nail plates. ${ }^{2}$ It is an underdiagnosed condition with different etiologies hypothesized, such as genetic factors with possible autosomal-dominant transmission and extrinsic factors. ${ }^{3}$ One proposed mechanism of pathogenesis is desynchronization during growth of the nail and distal phalanx of the hallux, leading to larger nail plates that grow laterally. ${ }^{4}$ Typical features associated with this disease are nail discoloration, nail plate thickening, and transversal grooves or ridges, none of which were seen in our patient. ${ }^{2}$

Children with nail-patella syndrome have dysplastic nails and associated bony abnormalities, such as absent patellae. 5 This syndrome results from an autosomaldominant mutation in the LIM homeobox transcription factor 1-beta gene, $L M X 1 B$, which is responsible for
A, A radiograph revealed an accessory distal phalanx of the left great toe. B, A similar smaller accessory distal phalanx on the right great toe.

dorsal-ventral patterning of the limb, as well as patterning of the nails, patellae and long bones, and even the kidney tubule. ${ }^{6}$ As such, patients with nail-patella syndrome have associated renal abnormalities. The findings in our patient were limited to the feet, making an underlying syndrome unlikely to be the cause.

First described in 1968 by Meadow, ${ }^{7}$ fetal hydantoin syndrome is a well-documented sequela in women taking phenytoin throughout pregnancy. Multiple malformations are possible, including cardiac defects, cleft lip/palate, digit and nail hypoplasia, abnormal facial features, mental disability, and growth abnormalities. ${ }^{8}$ The teratogenicity behind phenytoin results from reactive oxygen species that alter embryonic DNA, proteins, and lipids. ${ }^{9}$ The mother of this child was not on any seizure prophylaxis, eliminating it from the differential.

Congenital hypertrophy of the lateral nail fold is a defect of the soft tissue of the hallux leading to hypertrophy of the nail fold, commonly presenting with inflammation and pain ${ }^{10}$ possibly due to dyssynchronous growth between the soft tissue and nail plate. ${ }^{11}$ With this defect, a lip covering the nail plate is common, which was not seen in our patient.

As demonstrated in our patient, family history can help guide the diagnosis. Seven of 9 nonsyndromic forms of syndactyly are inherited in an autosomal-dominant fashion and range from mild presentations, as in our patient, to more severe deformations with underlying bone fusion and functional impairment. ${ }^{12}$ Polydactyly also often is expressed in an autosomal-dominant pattern, with up to $30 \%$ of patients having a positive family history. Polydactyly traditionally is classified by the location of the supernumerary digit as preaxial (radial), central, or postaxial (ulnar), and many further morphologic variations exist within these groups. Overall, preaxial polydactyly is relatively rare and represents $15 \%$ of polydactylies, with central and postaxial comprising the other $6 \%$ and $79 \%$, respectively. ${ }^{13}$ Delineation of the underlying anatomy may reveal ray duplications (digit and corresponding metacarpal or metatarsal bone), metatarsal variants, and duplicated phalanges that may be hypoplastic or deformed. Patients may report difficulty finding comfortable footwear, cosmetic concerns, and nail-related complications. Although not always required, surgical intervention may provide definitive treatment but can leave residual deformities in the surrounding altered anatomy; thus, orthopedic or plastic surgery consultations are critical in appropriately counseling patients.

\section{REFERENCES}

1. Ahmed H, Akbari H, Emanmi A, et al. Genetic overview of syndactyly and polydactyly. Plast Reconstr Surg Glob Open. 2017;5:e1549.

2. Catalfo P, Musumeci ML, Lacarrubba F, et al. Congenital malalignment of the great toenails: a review. Skin Appendage Disord. 2018;4:230-235.

3. Kus S, Tahmaz E, Gurunluoglu R, et al. Congenital malalignment of the great toenails in dizygotic twins. Pediatr Dermatol. 2005;22:434-435.

4. Chaniotakis I, Bonitsis N, Stergiopoulou C, et al. Dizygotic twins with congenital malalignment of the great toenails: reappraisal of the pathogenesis. J Am Acad Dermatol. 2007;57:711-715.

5. Witzgall R. Nail-patella syndrome. Pflugers Arch. 2017;469:927-936.

6. Dreyer SD, Zhou G, Baldini A, et al, Mutations in LMX1B cause abnormal skeletal patterning and renal dysplasia in nail patella syndrome. Nat Genet. 1998;19:47-50.

7. Meadow SR. Anticonvulsant drugs and congenital abnormalities. Lancet. 1968;2:1296.

8. Scheinfeld N. Phenytoin in cutaneous medicine: its uses, mechanisms and side effects. Dermatol Online J. 2003;9:6

9. Winn LM, Wells PG. Phenytoin-initiated DNA oxidation in murine embryo culture, and embryo protection by the antioxidative enzymes superoxide dismutase and catalase: evidence for reactive oxygen species-mediated DNA oxidation in the molecular mechanism of phenytoin teratogenicity. Mol Pharmacol. 1995;48:112-120.

10. Piraccini BM, Parente GL, Varotti E, et al. Congenital hypertrophy of the lateral nail folds of the hallux: clinical features and follow-up of seven cases. Pediatr Dermatol. 2000;17:348-351.

11. Martinet C, Pascal M, Civatte J, et al. Lateral nail-pad of the big toe in infants. apropos of 2 cases. Ann Dermatol Venereol. 1984;111:731-732.

12. Malik S. Syndactyly: phenotypes, genetics and current classification. Eur J Hum Genet. 2012;20:817-824.

13. Belthur MV, Linton JL, Barnes DA. The spectrum of preaxial polydactyly of the foot. J Pediatr Orthop. 2011;31:435-447. 